\title{
Optical Aberrations Caused by Transonic Attached Boundary Layers: Underlying Flow Structure
}

\author{
Aaron Buckner* \\ University of Notre Dame, Notre Dame, IN, 46556 \\ Stanislav Gordeyev ${ }^{\dagger}$ \\ University of Notre Dame, Notre Dame, IN, 46556 \\ and \\ Eric J. Jumper ${ }^{\ddagger}$ \\ University of Notre Dame, Notre Dame, IN, 46556
}

This paper is a continuation of an ongoing investigation of the optical distortions caused by a transonic attached turbulent boundary layer. To investigate the physical mechanisms of the optical aberrations, optical measurements with a high degree of temporal resolution using a Malley Probe were made along with unsteady surface pressure measurements and $X$-wire measurements at several different wall normal locations. It was found that wall pressure signals travel with the same convective speed as the optically aberrating structures, pointing toward pressure fluctuations in the outer part of the boundary layer as a primary cause for optical aberrations. Coherence lengths of flow structures are ${ }^{\S}$ obtained and compared to the coherence lengths of optically aberrating structures. Aperture effects on optical distortion are presented and discussed. The model for optical aberrations in turbulent sheared mean flows is developed and discussed in detail.

\section{Introduction}

When an otherwise planar optical wavefront is made to propagate through a variable index of refraction, turbulent flow field, the wavefront becomes aberrated, adversely affecting its far-field intensity pattern. This degraded far-field intensity pattern is undesirable for use in optical systems. The study of optical propagation through such flow fields is known as aero-optics ${ }^{1}$. Variable index flow fields come in many varieties, such as the mixing layer between two dissimilar index flow streams, and compressible boundary and free shear layers. The latter two scenarios are of great interest for the use of lasers on airborne platforms, specifically at Mach numbers greater than 0.5 and 0.3 , respectively.

The impact of optical aberrations on the performance of optical systems is usually reported as an average Strehl ratio, given by $S_{t}=\frac{I}{I_{0}}$, where $\mathrm{I}$ is the instantaneous on axis intensity, and $\mathrm{I}_{0}$ is the diffraction limited on axis intensity. Using the large aperture approximation, and given the time averaged $\mathrm{OPD}_{\mathrm{rms}}$ over the aperture, the Strehl ratio can be estimated by

$$
\overline{S_{t}}=\exp \left(-\frac{2 \pi \overline{O P D_{r m s}}}{\lambda}\right) \text {. }
$$

Studies $^{2}$ conducted in the late 1970s and early 1980s predicted that the $\mathrm{OPD}_{\mathrm{rms}}$ in a turbulent boundary layer was on the order of $0.1 \mu \mathrm{m}$. At the time, the affect of the turbulent boundary layer was thought to be inconsequential on the Strehl ratio, but as interest has shifted the propagation wavelength from $10.6 \mu \mathrm{m}$ to approximately $1 \mu \mathrm{m}$, the same OPD results give a reduction in the Strehl ratio of nearly $30 \%$.

Beginning in the early 1990s, free shear layers have been the subject of intense investigation. Much has been learned about the nature of optical aberrations due to fluid phenomenon due to this research, and

\footnotetext{
* Graduate Research Assistant, Department of Aerospace and Mech. Eng, AIAA Student Member.

${ }^{\dagger}$ Assistant Research Professor, Department of Aerospace and Mech. Eng., Member AIAA.

${ }^{\ddagger}$ Professor, Department of Aerospace and Mech. Eng., Fellow AIAA

Copyright (c) 2005 by A. Buckner, S. Gordeyev, E. Jumper. Published by the American Institute of Aeronautics and Astronautics, Inc. with permission.
} 
much of it can be applied to a turbulent boundary layer. The cause of the optical aberrations in shear layers was found to be the large scale structures that naturally "roll up". More particularly, it was found that the radial pressure gradients (and the associated density deficit) required to support the curvature of the structure were the cause of the optical aberrations. The source of the optical aberrations in a turbulent boundary layer was until recently, unknown, other than it is the result of fluctuating density.

Recent work $^{3}$ on the optically aberrating effects of turbulent boundary layers found that the optical aberrations scale with altitude, boundary layer thickness, and free stream Mach number. This work also suggested that coherent structures in the outer part of the boundary layer, and their associated pressure deficit, dominate the optical characteristics of optical aberrations. Large eddy simulations ${ }^{4}$ on the optical aberrations in a turbulent boundary layer show good agreement with experimental results, which also indicates that the optical signature of a turbulent boundary layer is dominated by the large scale motions. Several flow visualization studies ${ }^{5-7}$ have investigated coherent structures in turbulent boundary layers, and the prevailing theory is that coherent structures are the result of spanwise vortex structures that are created in the near wall region. These spanwise vortex structures are stretched in the streamwise direction into what is known as horseshoe or hairpin vortices. These structures, which are small in size, align themselves with one another, creating a large scale vortex packet. These vortex packets essentially lift away from the wall, creating large-scale coherent structures in the outer part of the boundary layer.

\section{Experimental Set-up}

All experiments were conducted at the Hessert Laboratory at the University of Notre Dame. The facilities were described in detail in Gordeyev at al. ${ }^{3}$ and an interested reader is referred to this reference for a complete discussion.

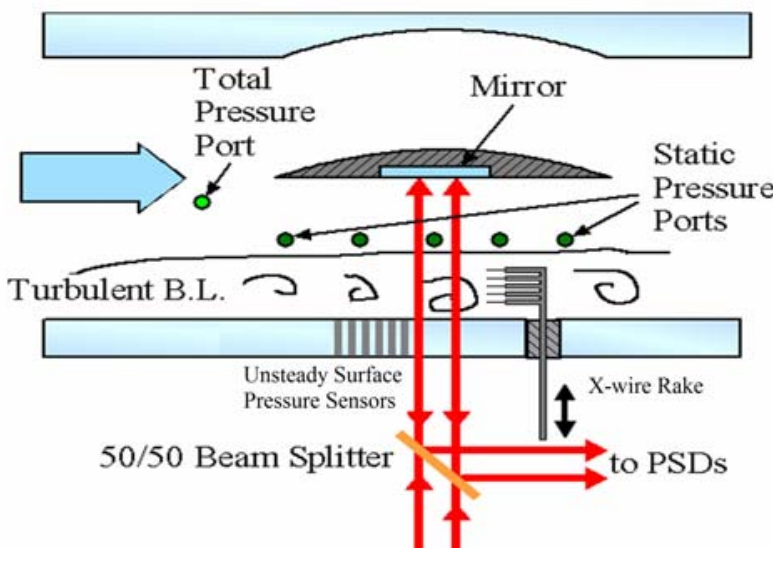

Figure 1. Schematic of the test section.

The test section was $9.9 \mathrm{~cm} \mathrm{X} 10.1 \mathrm{~cm}$ and was made of optically transparent plexiglas. A schematic of the test bottom of the section is presented in Figure 1. It was instrumented with static and dynamic pressure ports to monitor flow speed. The test section is also equipped with ports for unsteady surface pressure measurements. A schematic of the sensor layout can be found in Figure 2. The unsteady pressure sensors were piezo-resistive sensors manufactured by Kulite Semiconductor, Inc. and have good dynamic response up to $50 \mathrm{kHz}$. These sensors were used in conjunction with a commercial signal conditioner. Thomas, et. al. ${ }^{8}$ has shown that unsteady surface pressure sensors have the ability to detect dynamic events in a turbulent boundary layer.

The optical measurement technique chosen for this investigation was the Malley probe, which has been used extensively at the University of Notre Dame. Duffin, et al. ${ }^{9}$ describes this sensor and its operation, along with several other wavefront measurement techniques, in great detail. The Malley probe was chosen for this investigation for its high degree of temporal resolution and for its ease of use in the laboratory.

A single hot wire probe was used to measure traditional boundary layer profiles at the optical measurement location. The hot wire was used in conjunction with a commercial anemometer unit with a built in low pass filter. The signal was anti-alias filtered at $50 \mathrm{kHz}$, and the sampling frequency was

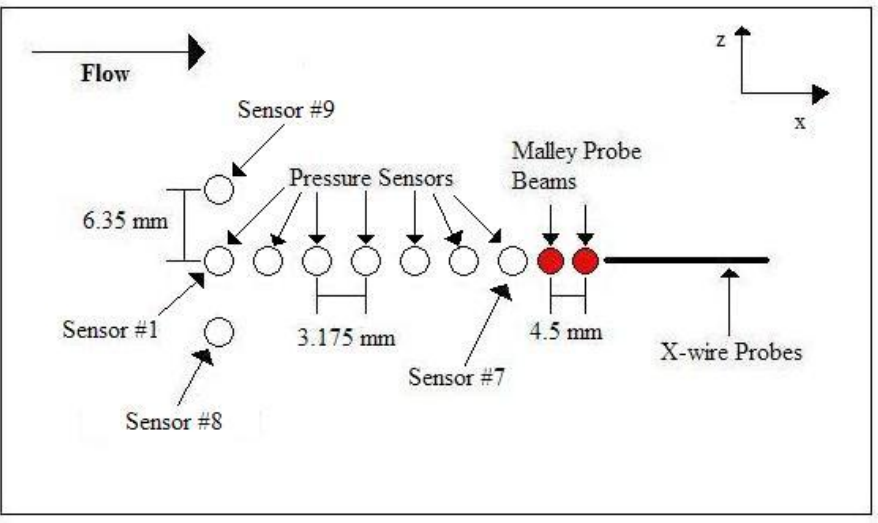

Figure 2. Schematic of the test section floor 
$100 \mathrm{kHz}$. The probe was calibrated in the mean flow (outside the boundary layer) at several Mach numbers ranging from 0.2 to 0.6. Boundary layer mean and rms velocity profiles were measured for two development lengths $(111.7 \mathrm{~cm}$ and $162.5 \mathrm{~cm}$ ), and at several different Mach numbers. The displacement thickness was calculated as

$$
\delta^{*}=\int_{0}^{\infty}\left(1-\frac{U(y)}{U_{\infty}}\right) d y
$$

A rake of four $\mathrm{x}$-wire probes was placed such that the probes were located immediately downstream of the optical measurement section. These probes were oriented in the wall normal direction (see Figure 1) to measure velocity fluctuations (simultaneous $\mathrm{u}-\mathrm{w}$ components) along the propagation path of the Malley probe beams. The four $x$-wire probes were located $3 \mathrm{~mm}, 10 \mathrm{~mm}, 15 \mathrm{~mm}$, and $20 \mathrm{~mm}$ from the wall. These $\mathrm{x}$-wire probes were used in conjunction with a commercial anemometer system. The probes were calibrated in the same wind tunnel described above, but with a shorter boundary layer development length. guaranteeing that all $\mathrm{x}$-wire probes are in the freestream flow and above the boundary layer. A two dimensional look up table assuming a King's law velocity dependence was used in the calibration of the $\mathrm{x}$ wires.

All data was acquired using an A/D system which was able to simultaneously sample 16 channels of data at $100 \mathrm{kHz}$ with no detectable lag between channels. The $\mathrm{x}$-wire signals and the Malley probe signals were anti-alias filtered at $50 \mathrm{kHz}$ using analog filtering circuits made in house. The unsteady pressure sensors exhibit a roll-off in their dynamic response at $50 \mathrm{kHz}$, so anti-alias filtering was not necessary with a sampling frequency of $100 \mathrm{kHz}$. All data was digitally high pass filtered at $0.5 \mathrm{kHz}$ in post processing to remove contamination from mechanical vibrations.

\section{Results}

\section{A. Fully turbulent boundary layer.}

The single hot wire boundary layer profile measurements were conducted to obtain mean and rms velocity profile measurements for a development length of $160 \mathrm{~cm}$ and a freestream Mach number of 0.47. Analysis of the boundary layer profiles showed a typical canonical turbulent boundary layer. A well defined log-law region is present, as shown in Figure 3. The slope in the log law region was defined as the reciprocal of the von Karman constant $(\mathrm{k}=0.39)$, and the friction velocity was iterated until this slope was achieved. The friction velocity that allows for agreement with the von Karman constant in the slope is $6.5 \mathrm{~m} / \mathrm{s}$. This confirms that the boundary layer was fully developed by the time it reached the measurement section.

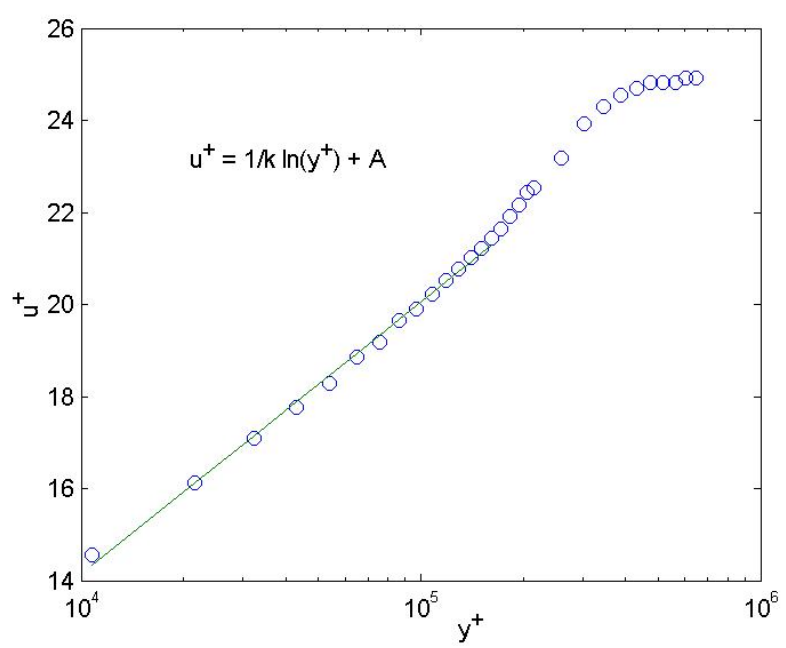

Figure 3. Velocity profile showing log law region.

\section{B. Streamwise Pressure Phase Correlation Results.}

Experimental results of phase correlations among streamwise arranged pressure sensors (relative to the pressure sensor \# 5) are presented in Figure 4. Phase plots clearly show convective nature of the underlying structure. Convective speeds obtained from these results are 0.7..0.88 of the freestream velocity. The phase scatter is due to fairly low values of pressure fluctuations near the wall, $\mathrm{p}_{\mathrm{rms}} / \mathrm{p}_{0} \sim 0.5 \%$, and mechanical vibration- and noise-related contaminations. Convective speeds based on the wall pressure data correspond quite well with the convective speed for the aberrating structures in the boundary layer of 0.8 of the freestream velocity obtained from Malley probe data, see Figure 5. Note that Malley probe phase results show much less scatter in phase slopes due to very sensitive nature of Malley probe, giving a high signal- 
to-noise ratio. High and similar convective speeds obtained from the pressure and Malley probe data further suggest the pressure "well' structure in the outer part of the boundary layer as a main cause of optical distortions, as proposed in Gordeyev at $\mathrm{al}^{3}$.
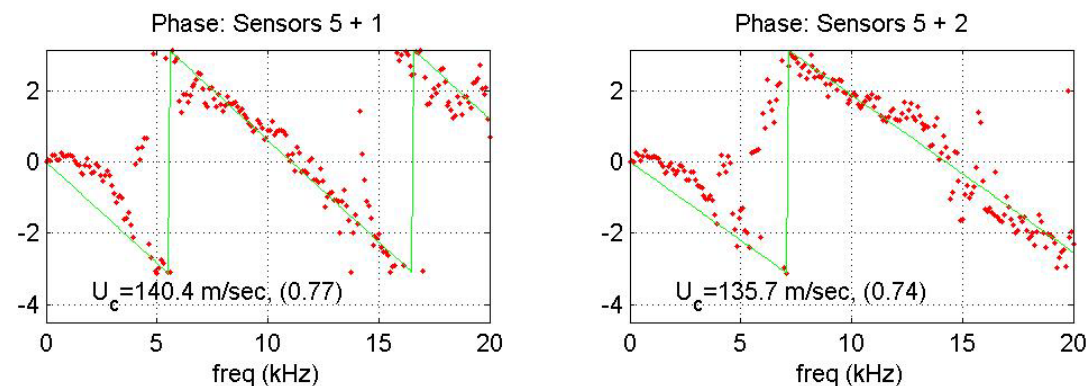

Phase: Sensors $5+3$

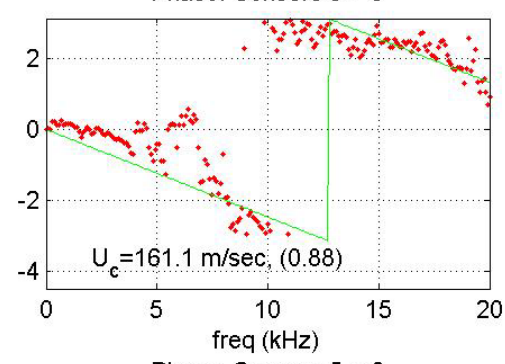

Phase: Sensors $5+4$

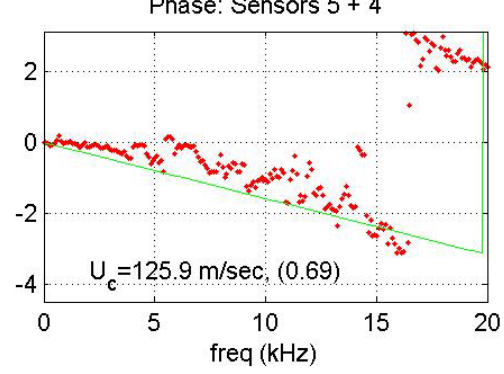

Phase: Sensors $5+6$

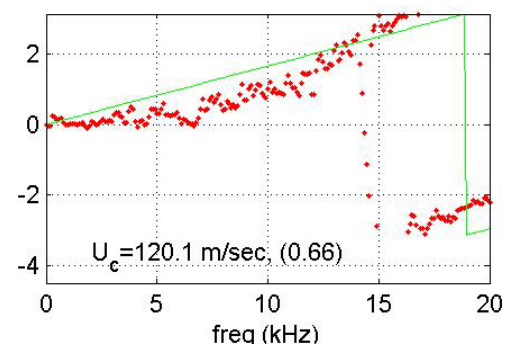

Phase: Sensors $5+7$

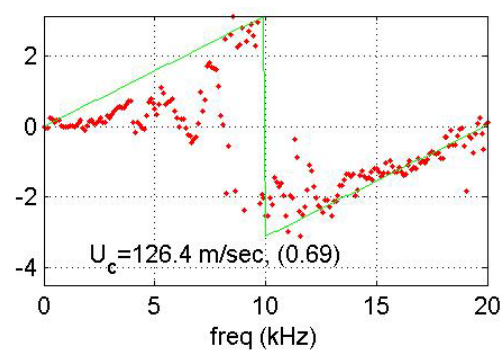

Figure 4. Phase correlations for 7 streamwise pressures sensors. Relative convective speeds are given in parenthesis. $M_{\text {free }}=\mathbf{0 . 5 4}$.

\section{Streamwise correlation lengths.}

Time series of wall pressures and OPD can be used to extract streamwise correlation scales. Time is converted to pseudo-streamwise coordinate $\mathrm{x}$ by the Taylor's frozen field assumption, $x=-t U_{c}$. A value of 0.8 of the freestream speed, as in Figure 5, was used for the connective speed $U_{c}$. Results for pressure signals are given in Figure 6. The streamwise correlation length $\Lambda_{x}^{p}$ is defined as a location of the first minima is the auto-correlation and it is $\Lambda_{x}^{p}=$ $1.2 \delta^{*}$ for $\delta^{*}=4.5 \mathrm{~mm}$.

Similarly, the pseudo-streamwise correlation length can be found from temporal evolution of $\mathrm{OPD}(\mathrm{t})$. Results are presented in Figure 7 for two different boundary layer thicknesses. The streamwise correlation OPD-

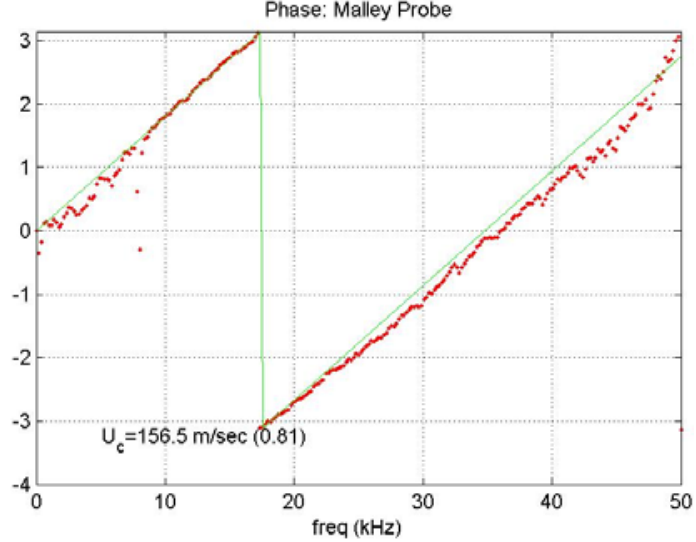

Figure 5. Malley probe phase correlation results, $M_{\text {free }}=0.57$.

based length $\Lambda_{x}^{O P D}$ is and $2 \delta^{*}$ for thick boundary layer. As discussed in Thomas et $\mathrm{al}^{8}$, there is no reason why the pressure footprint should correspond exactly to the structure passing above the surface. In fact, if 
there is to be a relationship, the pressure correlation length should be less than that of the concomitant coherent structure; the pressure coherence length meets this requirement. It is important to note that the structure size is 2 times bigger than the streamwise correlation length, giving the structure size to be $\sim 4 \delta^{*}$.

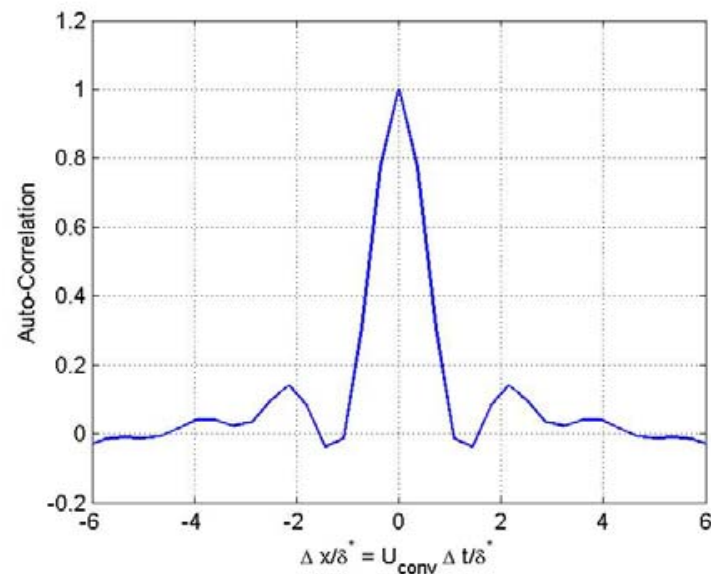

Figure 6. Pseudo-streamwise wall pressure normalized auto-correlation, $\delta^{*}=4.5 \mathrm{~mm}, \mathrm{M}_{\text {free }}=\mathbf{0 . 5 4}$.

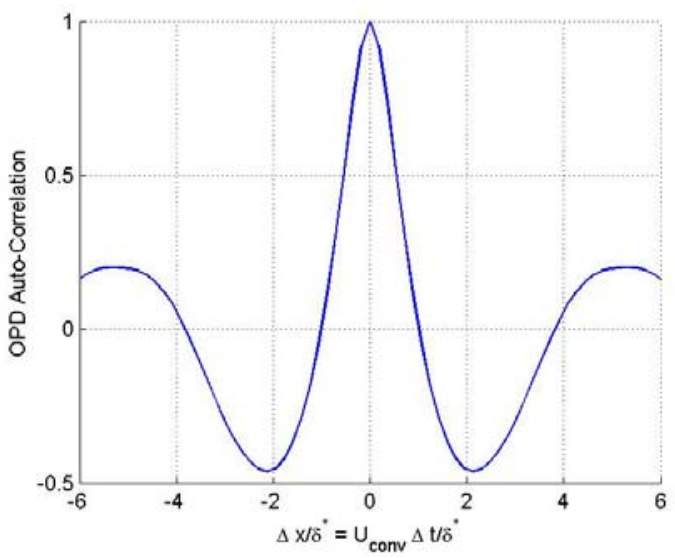

Figure 7. Pseudo-streamwise wall OPD normalized auto-correlation, $\delta^{*}=4.5 \mathrm{~mm}, \mathrm{M}_{\text {free }}=\mathbf{0 . 5}$.

\section{Spanwise Pressure Coherence Length.}

Spanwise unsteady surface pressure data were collected to measure the coherence length of structures in the spanwise direction. The normalized correlation coefficient was computed between all three spanwise unsteady pressure sensors. The experimental results are presented in Figure 8. An exponential fit of the form, $\rho(z, \Delta z)=\exp \left(-\Delta z / \Lambda_{z}\right)$, was performed on the three correlation coefficient functions and curve fits are also presented in Figure 8 as dashed lines. The average spanwise correlation length was found to be $\Lambda_{\mathrm{z}}=1.13 \delta^{*}$.

\section{E. Aperture effects.}

To investigate aperture effects, the experimentally-obtained OPD were apertured at different aperture sizes, $A_{\mathrm{p}}$, and, after removing a mean tilt from each frame, the residual $\operatorname{OPD}_{\text {rms }}\left(A_{\mathrm{p}}\right)$ was computed. The results are presented in Figure 9. For small aperture sizes the main optical distortions inside the aperture are mainly tilt; after removing tilt from the optical signal the residual $\mathrm{OPD}_{\mathrm{rms}}$ is

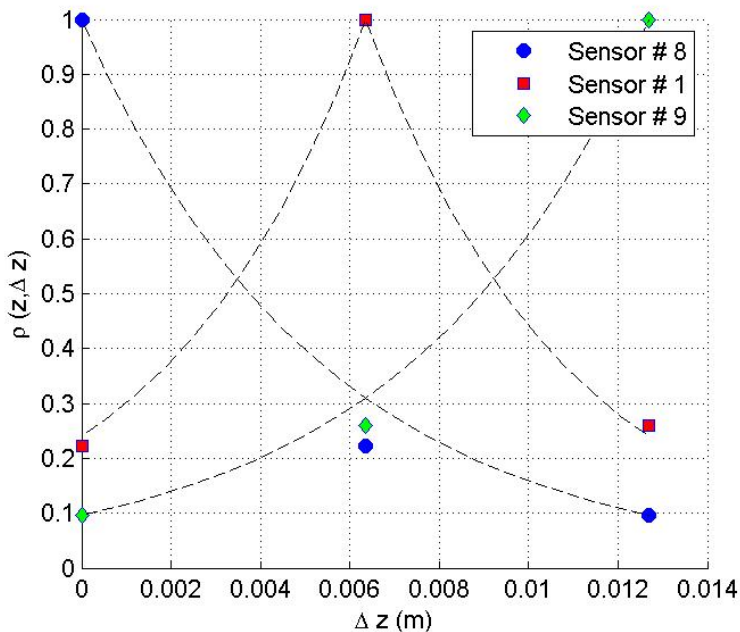

Figure 8. Spanwise pressure normalized correlation $\rho(\mathrm{z}, \Delta \mathrm{z})$; symbols are experimental results, dashed lines are exponential curve-fits. $\delta^{*}=4.5 \mathrm{~mm}, \mathrm{M}_{0}=0.57$. smaller than unapertured $\mathrm{OPD}_{\text {rms. }}$. When

the aperture size becomes sufficiently larger compared to the boundary layer thickness $\left(\sim 5-6 \delta^{*}\right)$, the $\mathrm{OPD}_{\mathrm{rms}}\left(A_{\mathrm{p}}\right)$ approaches the unapertured $\mathrm{OPD}_{\mathrm{rms}}=2.410^{-5} \rho / \rho_{\mathrm{SL}} M^{2} \delta^{*}$ measured in Gordeyev at al. ${ }^{3}$.

These important results can be used to estimate level of optical aberrations caused by turbulent boundary layer as a function of altitude, speed, boundary layer thickness and aperture sizes. In addition, these apertured results confirm the inferred structure sizes from the correlation-length data. Notice that $80 \%$ 
of the aberrating effect of the boundary layer is captured at an aperture size of $4 \delta^{*}$, consistent with a structure size of twice the coherence length reported in the earlier section.

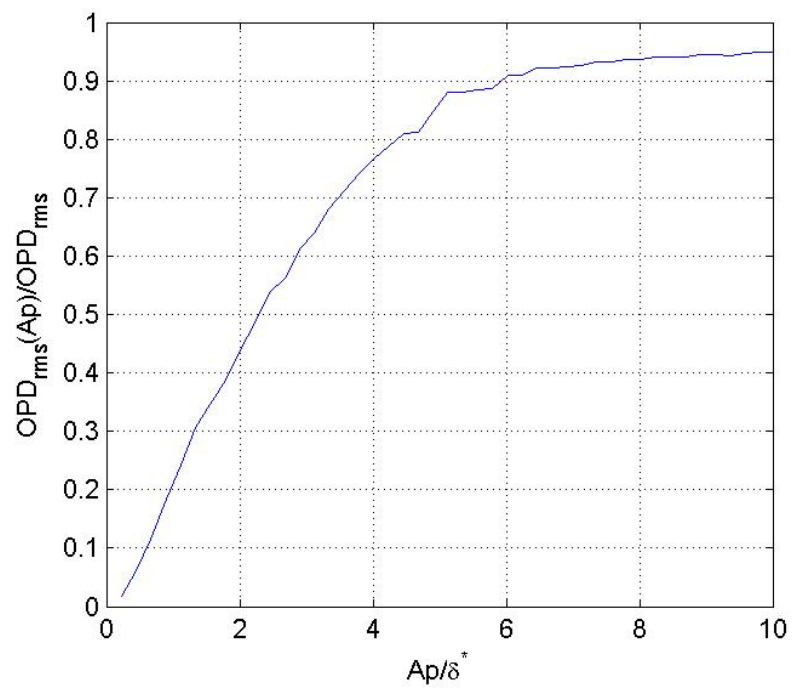

Figure 9. Aperture effects on OPDrms for turbulent boundary layers.

\section{Scaling arguments for sheared flows.}

In the previous paper ${ }^{3}$ a simple model of optical aberration caused by a traveling 2-D vortex was developed. Let us extend this theory to vortical structure moving in a sheared flow. Since pressure is a quadratic function of velocity field, the vortex - non-uniform sheared flow interaction will modify pressure distribution inside a vortical structure and change resulting optical distortions.

By definition, the Optical Path Length, OPL is proportional to an integral of density fluctuations $\rho^{\prime}(x, y)$ along a ray,

$$
O P L(x)=\int n(x, y) d y=\int n_{0}\left(1+K_{G D}\left(\rho-\rho_{0}\right) / n_{0}\right) d y=K_{G D} \int \rho^{\prime}(x, y) d y+C,
$$

where $K_{D G}$ is a Gladstone-Dale constant.

Assuming that density fluctuations are small relative to an freestream density $\rho_{0}, \rho<<\rho_{0}$, they can be expressed in pressure fluctuations $p^{\prime}$ as $p^{\prime} / p_{0}=\gamma\left(\rho^{\prime} / \rho_{0}\right)$ using the isentropic relation $p / p_{0} \sim\left(\rho / \rho_{0}\right)^{\gamma}$. Here and everywhere below the index " 0 ” defines freestream quantities. From the x-component momentum equation, pressure fluctuations can be expressed in terms of the velocity field, $\vec{u}(\vec{x}, t)=\vec{U}(\vec{x})+\vec{u}$ ' $(\vec{x}, t)$,

$$
-\frac{1}{\rho_{0}} \frac{\partial p^{\prime}}{\partial x}=\frac{\partial(u u)}{\partial x}+\frac{\partial(u v)}{\partial y}+\frac{\partial(u w)}{\partial z}
$$

Integrating the equation along the $y$-direction and applying boundary conditions $u^{\prime}=0$ at infinities or at the wall and than integrating along the x-direction and, finally we obtain the following expression,

$$
-\frac{1}{\rho_{0}} \int p^{\prime} d y=\int\left(U+u^{\prime}\right)^{2} d y+\frac{\partial}{\partial z} \iint u w d y d x \text {. }
$$

Assuming 2-D flow, $w=0$, a homogeneous flow in the x-direction, $U(x, y)=U(y)$ and a relatively weak turbulent flow, $u^{\prime}<<\mathrm{U}$, and also recognizing that $\gamma p_{0} / \rho_{0}=a_{0}^{2}$, where $a_{0}$ is a freestream speed of sound, the OPL can be re-written in terms of the u-velocity component as

$$
O P L(t)=-\frac{K_{G D} \rho_{0}}{a_{0}^{2}} \int\left(U^{2}(y)+2 U(y) u^{\prime}(y, t)+u^{\prime 2}(y, t)\right) d y \approx A-2 \frac{K_{G D} \rho_{0}}{a_{0}^{2}} \int U(y) u^{\prime}(y, t) d y
$$

and the equation for $\mathrm{OPD}(t)$ becomes (square brackets denote time averaging),

$$
O P D(t)=O P L(t)-\langle O P L(t)\rangle=-2 \frac{K_{G D} \rho_{0}}{a_{0}^{2}} \int U(y) u^{\prime}(y, t) d y \cdot
$$


Let us find the expression for $\mathrm{OPD}_{\mathrm{rms}}$. Squaring OPD and applying time averaging, we get the following,

$$
\begin{aligned}
& O P D_{r m s}^{2}=\left\langle O P D^{2}(t)\right\rangle=\left(2 \frac{K_{G D} \rho_{0}}{a_{0}^{2}}\right)^{2} \iint U(y) U\left(y^{\prime}\right)\left\langle u^{\prime}(y, t) u^{\prime}\left(y^{\prime}, t\right)\right\rangle d y d y^{\prime} \approx \\
& \left(2 \frac{K_{G D} \rho_{0}}{a_{0}^{2}}\right)^{2} \int U^{2}(y) u_{r m s}^{2}(y)\left(2 \Lambda_{y}^{u}(y)\right) d y
\end{aligned}
$$

where $\Lambda_{y}^{u}(y)=\int_{0}^{\infty} \frac{\left\langle u^{\prime}(y, t) u^{\prime}\left(y^{\prime}, t\right)\right\rangle}{u_{r m s}^{2}(y)} d y^{\prime}=\int_{0}^{\infty} \rho\left(y, y^{\prime}\right) d y^{\prime}$ is the $u$-correlation length in the y-direction.

In deriving equation (3) it was assumed that the mean velocity profile varies on a bigger scale than the correlation length. For a boundary layer it is not true near the wall where the mean velocity profile changes significantly, but in the area of interest (the outer portion of the boundary layer) it changes slowly relative to the u-correlation scale. Thus, knowing the mean velocity, fluctuating velocity and y-correlation length along the $y$-direction, it is possible to estimate the level of optical distortions $\mathrm{OPD}_{\text {rms. }}$.

(a)

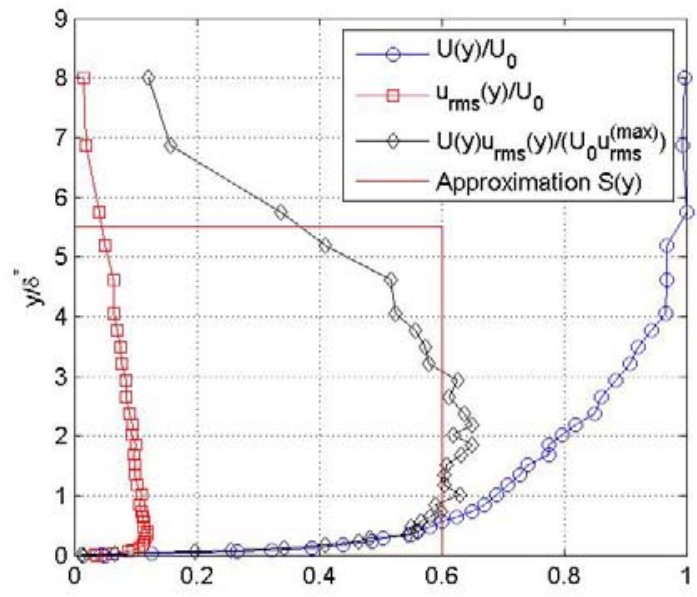

(b)

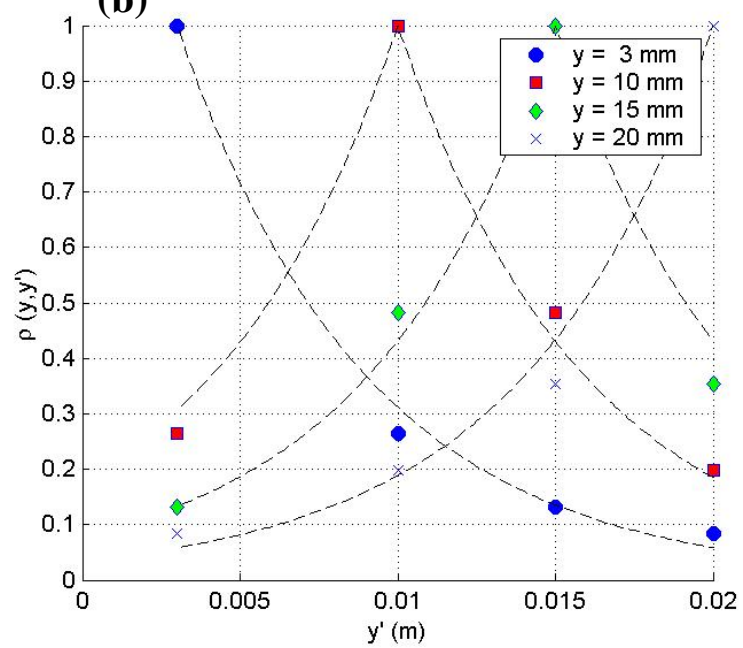

Figure 10: (a) Mean $U(y)$, fluctuation component $u_{\mathrm{rms}}(y)$, product $U(y) \mathrm{u}_{\mathrm{rms}}(y)$ and $\mathrm{S}(\mathrm{y})$ profiles for the boundary layer; (b) u-component normalized correlations $\rho\left(y, y^{\prime}\right)$, symbols are experimental results, dashed lines are exponential curve-fits. $\delta^{*}=4.2 \mathrm{~mm}, \mathrm{M}_{0}=0.57$.

Let us apply this estimation to the fully developed transonic turbulent boundary layer. Mean and fluctuating velocity profiles measured using the single hot-wire are presented in Figure 10(a). The product $U(y) u_{\text {rms }}(y)$ is also presented in Figure 10(a). The product is fairly constant inside the boundary layer $y / \delta^{*}<5.5$ and quickly drops off to zero outside of it. Note that although the maximum value of $u_{\text {rms }}$ occurs near the wall, the maximum value of the $U(y) u_{\mathrm{rms}}(y)$ product is well inside of the outer part of the boundary layer at $y / \delta^{*}=2$, where $U(y) / U_{0}=0.8$. Again, this emphasizes the importance of the outer part of the boundary layer for optical aberrations. For estimation purposes we will approximate the velocity product with a simplified piecewise-constant function $S(y), S(y)=0.6 U_{0} u_{r m s}^{(\max )}$ for $y / \delta^{*}<5.5$ and $S(y)=0$ otherwise, as shown in Figure 10(a).

Results of the u-velocity correlation using the 4-hot-wire rake are presented in Figure 10(b). For estimation purposes we assume that $\rho\left(y, y^{\prime}\right) \approx \exp \left[-\left|y-y^{\prime}\right| / \Lambda_{y}^{u}(y)\right]$. The least square curve fitting of the data are shown in Figure 10(b) in dashed lines and gives a fairly constant value of the u-correlation length across the boundary layer of $\Lambda_{y}^{u}=1.33 \delta^{*}$. Thus the ratio of the boundary layer thickness to the ucorrelation scale is $5.5 / 1.33=4.1$ and the assumption of the slowly varying mean velocity profile, used in deriving (3) is reasonably true.

Substituting everything into (3), we get 


$$
O P D_{r m s}^{2} \approx\left(2 \frac{K_{G D} \rho_{0}}{a_{0}^{2}} U_{0} u_{r m s}^{(\max )}\right)^{2} \Lambda_{y}^{u} \int\left(2 S^{2}(y)\right) d y=\left(2 \frac{K_{G D} \rho_{0}}{a_{0}^{2}} U_{0} u_{r m s}^{(\max )} \delta^{*}\right)^{2}\left(2 \cdot 0.6^{2} \cdot 1.33 \cdot 5.5\right)
$$

or

$$
O P D_{r m s} \approx 4.6 \frac{K_{G D} \rho_{0}}{a_{0}^{2}} U_{0}^{2} \frac{u_{r m s}^{(\max )}}{U_{0}} \delta^{*}=4.6 K_{G D} \rho_{0} M_{0}^{2} t^{(\max )} \delta^{*}=11.7 \cdot 10^{-5} \rho_{0} / \rho_{S L} M_{0}^{2} \delta^{*}
$$

where $t^{(\max )}=u_{r m s}^{(\max )} / U_{0}$ is the relative turbulence intensity in the boundary layer.

Comparing the estimated $\mathrm{OPD}_{\mathrm{rms}}$ (4) with the experimental results presented in earlier in Gordeyev et al. ${ }^{3}$, this estimation over-predicts optical aberrations by a factor of 4 . It is not clear why the coefficient is off by 4, surely many of the cavalier assumptions contribute; however, it is also the case that the 2-D assumption may be a contributing factor, since pressure wells might be expected to be dipper in the 2-D flow than in 3$\mathrm{D}$ case. Of course, these modeling results are preliminary and should be considered as a first step in the future more complete analysis.

It is important to note that the presented model and simple moving vortex model developed in Ref. 3 give the same experimentally confirmed functional dependence $\mathrm{OPD}_{\mathrm{rms}} \sim \rho \mathrm{M}^{2} \delta$; the simple model underpredicts the experimentally obtained numerical coefficient (ref) by a factor of 2 , since it does not take into account the interaction between the vortical structure and the shear-like mean velocity profile.

\section{Conclusions and Discussions}

Additional experimental studies beyond those of Ref. 3 have been carried out on the "opticallyaberrating, fully-developed transonic turbulent boundary layer at $\mathrm{M}=0.5 . .0 .6$ using wall pressure sensors, a rake of $\mathrm{x}$-wire probes and a Malley probe, which is capable of accurately measuring levels of optical distortions in turbulent flows. The results demonstrate a clear association between the unsteady pressure footprint on the wall and the convection of coherent aberrating structures in the boundary layer. This association was established first in the shared convection velocities of the pressure-producing and aberrating structures in the boundary layer, approximately 0.8 of the freestream speed. This association and shared convective velocity indicates that common structures produce the pressure fluctuations, and these common coherent (vortical) structures are present in the outer part of the boundary layer. The streamwise correlation lengths of these aberrating structures based on the wall pressure data are approximately 1.2 times of the displacement thickness $\delta^{*}$. For reasons discussed in the paper, these pressure correlation lengths should be somewhat smaller than the associated structures convecting in the outer portion of the boundary layer; indeed, the streamwise correlation lengths for the optical signals yield a somewhat higher value for the coherence length of the actual aberrating extent of the coherent, vortical structures of $2 \delta^{*}$, yielding the structure size to be approximately twice of that. From this definite association between the pressure footprint and the aberrating structure, we are able to infer the approximate spanwise coherence length of the aberrating structures by examining the unsteady pressure crosscorrelation-derived coherence lengths; these coherence lengths were found to be approximately the same as the streamwise coherence length, $\sim 1.1 \delta^{*}$.

A simple model of optical distortions in turbulent flows was developed, which is in fact a further improvement of the model presented earlier in Ref. 3, for the case of vortical structures in non-uniform sheared mean flows. Both models are in agreement on a functional dependence of optical aberrations OPDrms which is proportional to a freestream density, square of the freestream Mach number and the displacement thickness, $\mathrm{OPD}_{\mathrm{rms}} \sim \rho \mathrm{M}^{2} \delta$; these findings are really useful when scaling optical data to different flow conditions. Also aperture effects on OPDrms are presented and discussed.

\section{Acknowledgments.}

These efforts were sponsored by the Air Force Office of Scientific Research, Air Force Material Command, USAF, under Grant Number F49620-03-1-0019. The U.S. Government is authorized to reproduce and distribute reprints for governmental purposes notwithstanding any copyright notation thereon. 


\section{References}

${ }^{1}$ Jumper, E. J., and Fitzgerald, E. J., “Recent Advances in Aero-Optics,” Progress in Aerospace Sciences, Vol. 37, 2001, pp. 299-339.

2 Rose, W. C., "Measurements of Aerodynamic Parameters Affecting Optical Performance,” Air Force Weapons Laboratory Final Report, AFWL-TR-78-191, May 1979.

${ }^{3}$ Gordeyev, S., Jumper E .J., Ng, T. T., and Cain, A. B., "Aero-Optical Characteristics of Compressible, Subsonic, Turbulent Boundary Layers,” AIAA Paper 2003-3606, June 2003..

${ }^{4}$ Tromeur, E., Garnier, E., Sagaut, P., Basdevant, C., "Large eddy simulations of aero-optical effects in a turbulent boundary layer,” Journal of Turbulence, Vol. 4, 2003, pp.

${ }^{5}$ Christensen, K. T., and Adrian, R. J., "The Velocity and Acceleration Signatures of Small-Scale Vortices in Turbulent Channel Flow," Proceedings of the Second International Symposium on Turbulence and Shear Flow Phenomenon, June 2001.

${ }^{6}$ Ganapathisubramani, B., Longmire, E. K., and Marusic, I., "Characteristics of vortex packets in turbulent boundary layers,” Journal of Fluid Mechanics, Vol. 478, 2003, pp. 35-46.

7 Dargahi, B., "Generation of Coherent Structures in Turbulent Boundary Layers," Journal of Engineering Mechanics, Vol. 123, No. 7, 1997, pp. 686-695.

8 Thomas, F. O.; Putnam, C. M., and Chu, H. C., "On the mechanism of unsteady shock oscillation in shock wave/turbulent boundary layer interactions,” Experiments in Fluids, Vol. 18, 1994, pp. 70-75.

${ }^{9}$ Duffin, D. A., Gordeyev, S., and Jumper, E. J., "Comparison of Wavefront Measurement Techniques on a TwoDimensional Heated Jet,” AIAA Paper 2004-2406, June 2004. 